\title{
A case report of right upper lung adenosquamous carcinoma resection following neoadjuvant targeted therapy
}

\author{
Nan Zhang^, Shuxing Chen, Liren Guo, Xing Chen \\ Department of Thoracic Surgery, Fuzhou Pulmonary Hospital of Fujian, Fuzhou, China \\ Correspondence to: Nan Zhang. No.2 Hubian Road, Cangshan District, Fuzhou 350000, China. Email: zhangnan235@126.com.
}

\begin{abstract}
The patient was a middle-aged male smoker who had space-occupying lesions in the right upper lung, Positron emission tomography-computed tomography (PET-CT) suggested right upper lung cancer with multiple mediastinal lymph node metastases. Endobronchial ultrasound-guided transbronchial lung biopsy (EBUS-GS-TBLB) performed in the bronchus of the right apical segment confirmed the lesion as squamous cell carcinoma. Neoadjuvant therapy was planned. Because genetic testing revealed the epidermal growth factor receptor (EGFR) L858R mutation, the possibility of adenosquamous carcinoma was considered. The patient was clinically diagnosed with right upper lung squamous cell carcinoma c-T3N2M0, stage IIIB, and PS 1 point. Dacomitinib was selected for the targeted therapy. Eight weeks after the initiation of treatment, efficacy was assessed as a partial response (PR), suggesting a possibility of R0 resection. After sufficient communication with the patient and his family members, on July 1, 2020, thoracoscopic radical resection of the right upper lung cancer was performed under general anesthesia. Postoperative pathology confirmed adenosquamous carcinoma, of which $80 \%$ were adenocarcinoma and $20 \%$ were squamous cell carcinoma; no tumor thrombus was seen in the interstitial vessels; and the tumor did not invade the visceral pleura. There was no cancerous infiltration in the bronchial stump of the right upper lung. The following were the results for lymph nodes submitted for examination: L2 (0/1), L4 (0/4), L7 (0/3), L8 (0/7), L10 $(0 / 1)$, L11 (0/2) and L11 (frozen 0/1) showed no cancer metastasis. The postoperative diagnosis was as follows: adenosquamous carcinoma of the right upper lung p-T2N0M0, stage Ib. After surgery, 4 cycles of the GC (gemcitabine + carboplatin) chemotherapy regimen were given, with continued targeted therapy recommended for 2 years. The patient has been followed-up and is in good condition.
\end{abstract}

Keywords: Adenosquamous carcinoma; L858R mutation; neoadjuvant targeted therapy; radical resection of lung cancer

Submitted Feb 26, 2021. Accepted for publication Apr 21, 2021.

doi: $10.21037 /$ apm-21-868

View this article at: http://dx.doi.org/10.21037/apm-21-868

\section{Introduction}

At present, there are still controversies regarding the treatment of operable locally advanced non-small cell lung cancer. Neoadjuvant therapy combined with surgery is one of the main treatment options, but there are numerous modes (chemotherapy alone, sequential chemoradiotherapy, concurrent chemoradiotherapy, concurrent chemoradiotherapy after chemotherapy, targeted therapy and immunotherapy), each with its own advantages and disadvantages. The diagnosis and treatment guidelines encourage patients to participate in relevant clinical trials. Neoadjuvant immunotherapy is currently a hot topic, but the roles of neoadjuvant targeted therapy should not be ignored.

This case involved a patient with adenosquamous carcinoma in the right upper lung (c-T3N2M0) who

\footnotetext{
$\wedge$ ORCID: 0000-0002-1364-5532.
} 

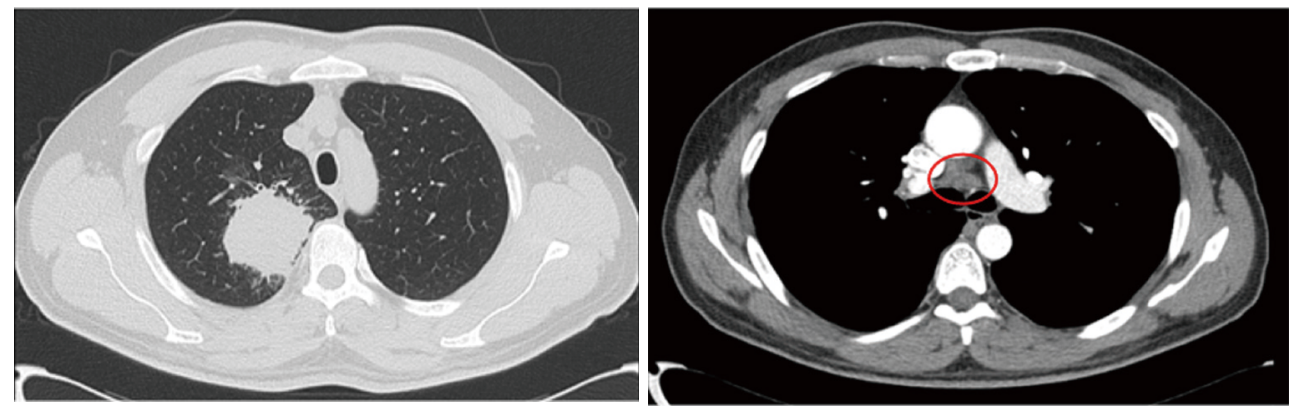

Figure 1 2020.4.8 Chest CT indicated a space-occupying lesion in the right upper lung, with mediastinal lymphadenopathy, and the unevenness was enhanced after contrast enhancement. CT, computed tomography.

was treated by our treatment group. The EGFR L858R mutation was detected in this patient. After oral administration of dacomitinib for 8 weeks, the disease was assessed to be significantly relieved. Subsequently, thoracoscopic radical resection of the right upper lung cancer was performed to provide an opportunity for radical treatment.

We present the following case in accordance with the CARE Reporting Checklist (available at http://dx.doi. org/10.21037/apm-21-868).

\section{Case presentation}

\section{Clinical data}

A 42-year-old male patient was admitted to our hospital on April 7, 2020, with the chief complaint of "chronic cough for over 2 months". Chest computed tomography (CT) taken at another hospital showed a mass in the upper lobe of the right lung with cavitation, measuring approximately $5.3 \mathrm{~cm} \times 5.5 \mathrm{~cm}$ in size, with mediastinal lymphadenopathy. The mass was considered to be a tumor, and the patient was referred to our hospital for further treatment. The patient had no special medical history and had been smoking for 20 years (1 pack/day). Upon physical examination, breath sounds in the right upper lung were weak, and dry or moist rales were not heard. Bilateral supraclavicular lymph nodes were not palpable. An immunology panel revealed the following: CEA, $25.34 \mathrm{ng} / \mathrm{mL}$; CA125, $40.63 \mathrm{kU} / \mathrm{L}$; CA153, $52.77 \mathrm{kU} / \mathrm{L}$; and SCC, $19.68 \mathrm{ng} / \mathrm{mL}$. Plain + enhanced chest CT was performed on April 8, 2020, with the following findings: part of the bronchial wall in the upper lobe of the right lung was irregularly thickened, the lumen was narrowed and obstructed, and a mass-like soft tissue shadow was visible in the surrounding area, measuring approximately $5.3 \mathrm{~cm} \times 5.1 \mathrm{~cm}$ in size, with uneven density. After contrast enhancement, the following findings were observed: the unevenness was enhanced, the margins were well-defined, lobes could be seen, lymph node shadows $(2 \mathrm{R}, 4 \mathrm{R} / \mathrm{L}, 7,8,10 \mathrm{R} / \mathrm{L}, 11 \mathrm{R})$ were visible in the mediastinum, with some of them being enlarged, and enhancement was visible (see Figure 1). On 12 April, 2020, whole body positron emission tomography-computed tomography (PET-CT) was performed, with the following findings: space-occupying lesions in the right upper lung and abnormal radioactive concentration in the $2 \mathrm{R}, 4 \mathrm{R}$, 7, 8 and $11 \mathrm{R}$ lymph nodes, with increased standardized uptake values (SUVs). The lesion was considered to be lung cancer with mediastinal lymph node metastasis, with no other metastasis found.

All procedures performed in studies involving human participants were in accordance with the ethical standards of the institutional and/or national research committee(s) and with the Helsinki Declaration (as revised in 2013). Written informed consent was obtained from the patient for publication of this manuscript and any accompanying images.

\section{Diagnosis and neoadjuvant targeted therapy}

To confirm the diagnosis, fiberoptic bronchoscopy was performed on April 14, 2020, and no abnormality of the tracheal lumen was observed (Figure 2). A heterogeneous echo was detected in the apical segment of the right upper lobe (RB1) using an ultrasound probe (Figure 3). EBUSGS-TBLB was performed, with the following pathology results: CK5/6 (3 +), P40 (3 +), P63 (3+), p53 (approximately $80 \%+)$, P16 (3 +), CK7 (+), and TTF-1 (-); "RB1a TBLB" indicated squamous cell carcinoma (Figure 4). The patient 


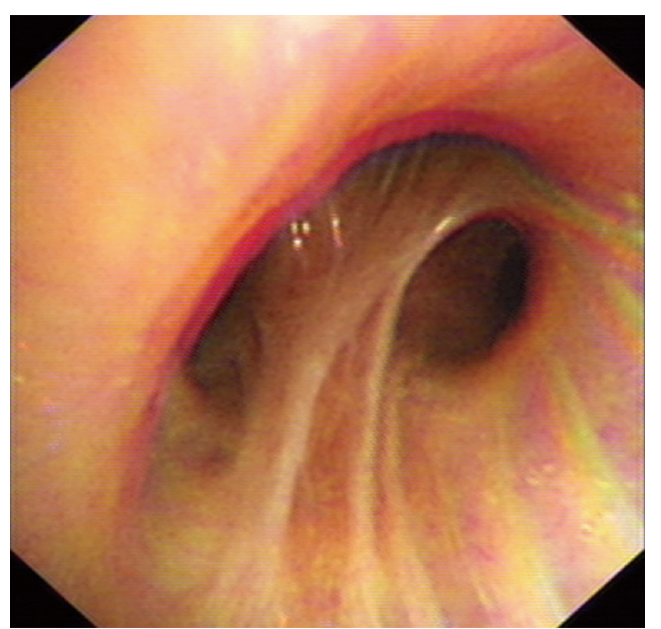

Figure 2 No abnormality in the bronchial orifice of the right upper lung was observed.

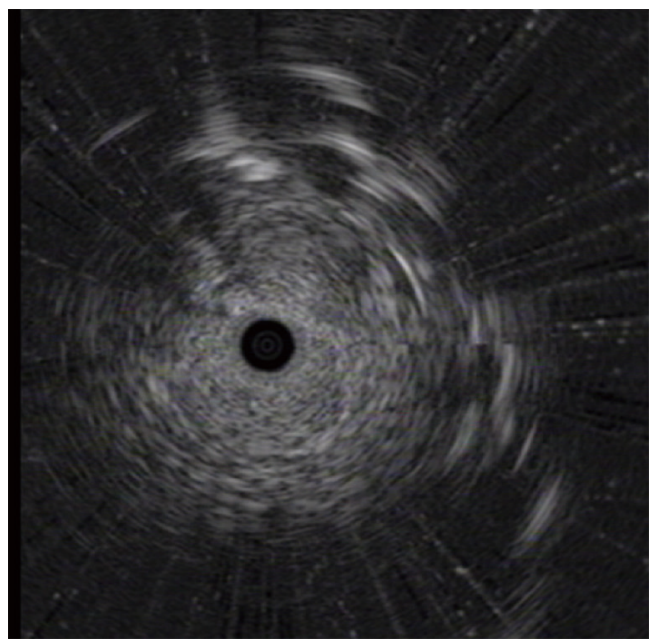

Figure 3 A heterogeneous echo pattern was observed in the ultrasound image of the right upper lung apex.

was clinically diagnosed with right upper lung squamous cell carcinoma c-T3N2M0, stage IIIB, and PS 1 point. Genetic testing was performed to evaluate the presence or absence of immunotherapy indications, with the following test results: PD-L1 TPS, 30\%; KRAS, ALK, BRAF, HER2, NRAS, PIK3CA, ROS1, and RET, wild-type; and EGFR, L858R mutation.

Considering that the rate of EGFR mutations in simple squamous cell carcinoma is very low $(<4 \%)$ and that the mixed adenocarcinoma component may not be detected in this patient due to tumor heterogeneity, it was speculated

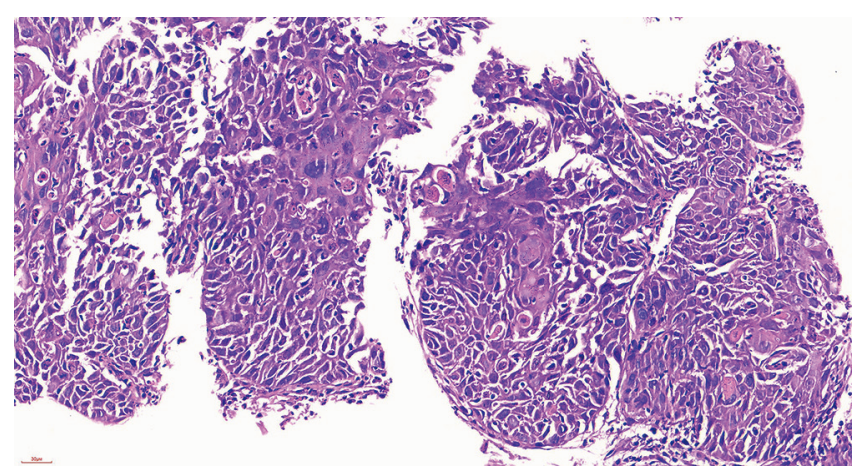

Figure 4 TBLB of the apical segment of the right lung revealed squamous cell carcinoma, HE staining, $\times 400$. TBLB, transbronchial lung biopsy.

that the patient may have adenosquamous carcinoma. Referring to the FLAURA study, the ARCHER 1050 study and the consensus of Professor Yilong $\mathrm{Wu}$ at the 17th Summit Forum on Lung Cancer, dacomitinib was selected for neoadjuvant targeted therapy. On April 24, 2020, the patient initiated treatment with dacomitinib (45 mg, orally, qd); additionally, thymalfasin was administered to boost immunity (1.6 mg, subcutaneous injection, twice a week). Efficacy was evaluated 2 months later.

\section{Assessment after targeted therapy}

On May 13, 2020, plain chest CT reexamination showed that compared with the CT performed in our hospital on April 8, 2020, part of the bronchial wall of the right upper lobe was irregularly thickened, lumen stenosis improved, a smaller mass-like soft tissue shadow (approximately $3.2 \mathrm{~cm} \times 3.0 \mathrm{~cm}$ ) was observed in surrounding areas, the current size was smaller, with an uneven density but clear margins, the lobes were visible, and lymph node shadows were visible in the mediastinum (some were enlarged, and some were smaller than before). On June 24, 2020, plain chest CT reexamination showed that compared with the CT performed in our hospital on May 13, 2020, part of the bronchial wall of the right upper lobe was irregularly thickened, lumen stenosis improved, a smaller masslike soft tissue shadow (approximately $2.6 \mathrm{~cm} \times 2.4 \mathrm{~cm}$, with an uneven density) was observed in surrounding areas, the inner irregular radiolucent shadow was smaller, and the margins were clear. The lobes were visible, and lymph node shadows $(2 \mathrm{R}, 4 \mathrm{R} / \mathrm{L}, 7,8)$ were visible in the mediastinum (some of them were smaller than 

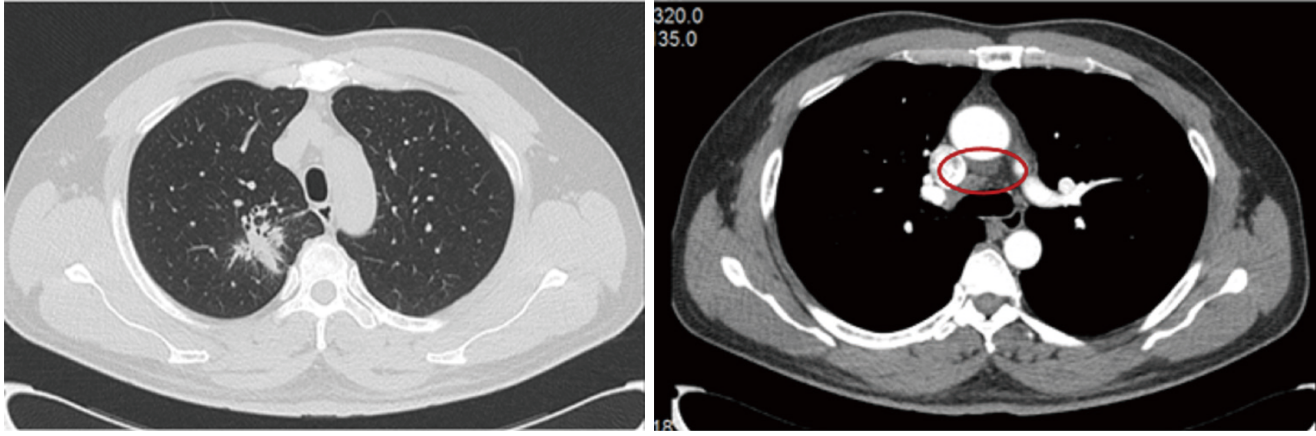

Figure 5 2020.6.24 Chest CT showed that the right lung tumor and mediastinal lymph nodes were significantly smaller after targeted therapy. CT, computed tomography.

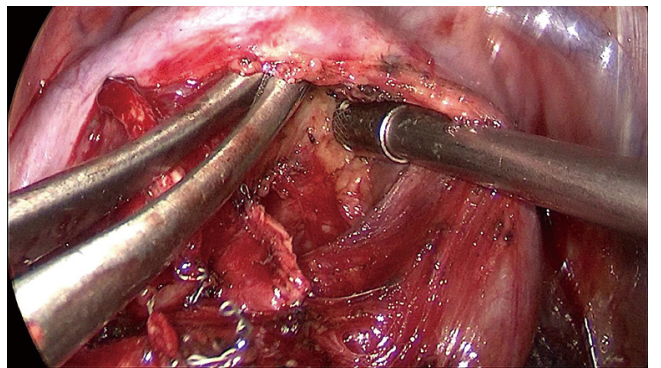

Figure 6 Dissection of the $2^{\text {nd }}$ and $4^{\text {th }}$ groups of lymph nodes.

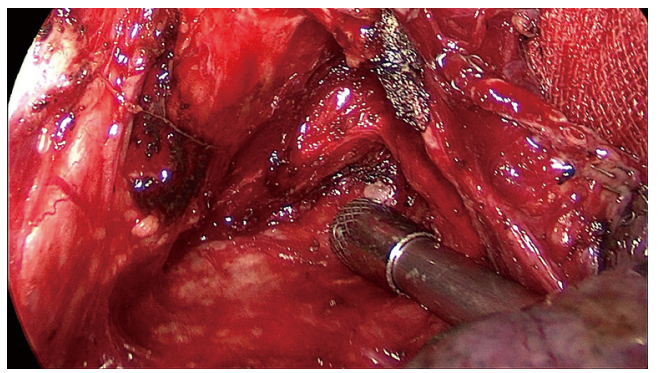

Figure 7 Dissection of the $7^{\text {th }}$ and $8^{\text {th }}$ groups of lymph nodes.

before) (Figure 5). On June 22, 2020, immunology panel reexamination revealed the following: CEA, $5.22 \mathrm{ng} / \mathrm{mL}$; CA125, $14.76 \mathrm{kU} / \mathrm{L}$; CA153, $21.05 \mathrm{kU} / \mathrm{L}$; and SCC, $4.40 \mathrm{ng} / \mathrm{mL}$. During treatment, the main adverse reaction was a grade I rash on the face. After 8 weeks of targeted therapy, the sizes of the tumor and mediastinal lymph nodes were significantly smaller, and the tumor indicators were substantially lower. Therefore, efficacy was assessed as PR. R0 resection was considered. After full communication with the patient and his family, we chose to perform thoracoscopic radical resection of the right upper lung cancer under general anesthesia.

\section{Surgery and recovery}

On July 1, 2020, the patient underwent thoracoscopic radical resection of the right upper lung cancer under general anesthesia. Intraoperative findings included slight adhesion between the right upper lung and the chest wall, incomplete development of a pulmonary fissure, no pleural effusion, a lesion in the right upper lung $(2.5 \times 2.5 \times 3 \mathrm{~cm})$ with moderate texture and poorly defined margins, and no pleural indentation on the surface; no abnormalities in the right middle and lower lobes; and enlarged lymph nodes visible between the lobes and in the mediastinum. The adhesions were separated, the right lower pulmonary ligament was freed, and the pleura was opened. The right upper pulmonary artery and vein and right upper pulmonary bronchus were dissected and separated, the right upper lobe was removed, and the $2 \mathrm{R}, 4 \mathrm{R}, 7,8,9,10 \mathrm{R}$, and $11 \mathrm{R}$ lymph nodes were dissected (Figures 6,7). The intraoperative pathological examination of frozen sections showed that the bronchial stump was negative and that there was no metastasis in the parabronchial lymph nodes of the right upper lobe. The patient was postoperatively rehydrated and provided analgesia, nebulization, and immune enhancement treatments; the patient recovered quickly (Figure 8). All drainage tubes were removed 2 days after the surgery, and the patient was discharged 5 days after the surgery.

\section{Postoperative diagnosis and subsequent treatment}

The postoperative pathology results were as follows: right upper lobectomy specimen - double label: TTF-1 (adenocarcinoma +), elastic fibers (-), CK7 (adenocarcinoma 
+), TTF-1 (adenocarcinoma +), NAPSINA (adenocarcinoma +), P40 (squamous cell carcinoma +), and CK5/6 (squamous cell carcinoma + ); right upper lobectomy specimen adenosquamous carcinoma (Figure 9), of which $80 \%$ were adenocarcinoma and $20 \%$ were squamous cell carcinoma; no tumor thrombus was seen in the interstitial vessels; and the tumor did not invade the visceral pleura. There was no cancerous infiltration in the bronchial stump of the right upper lung. The following were the results for lymph nodes submitted for examination: L2 (0/1), L4 (0/4), L7 (0/3), L8 (0/7), L10 (0/1), L11 (0/2) and L11 (frozen 0/1) showed no cancer metastasis. The postoperative diagnosis was as follows: adenosquamous carcinoma of the right upper lung p- $\mathrm{T}_{2} \mathrm{~N}_{0} \mathrm{M}_{0}$, stage Ib, which failed to achieve MPR according

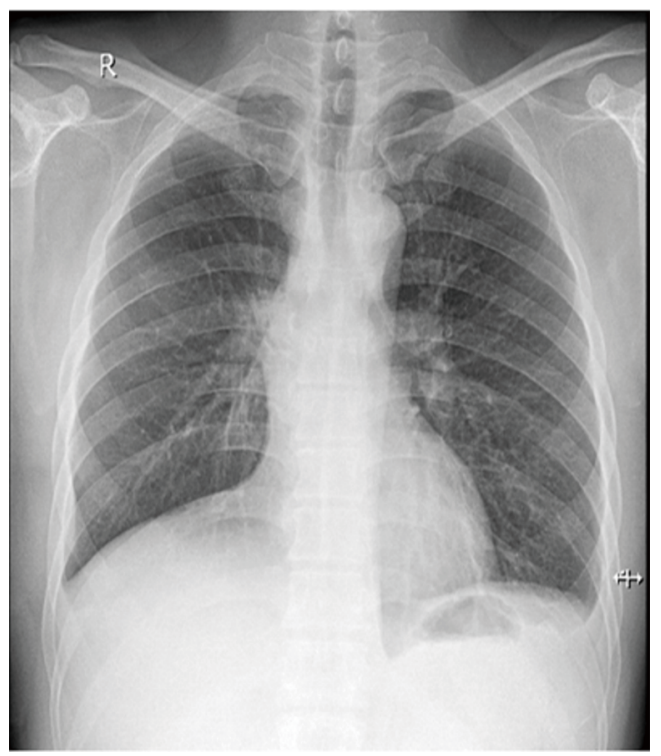

Figure 8 Chest X-ray on postoperative day 4. to the pathologist's reading of the film; the total tumor cells were reduced by approximately $30 \%$.

According to the diagnosis and treatment guidelines and referring to the ADAURA and EVAN studies, continued oral administration of dacomitinib was recommended for 2 more years. For the $20 \%$ squamous cell carcinoma in the tumor, the GC chemotherapy regimen was given for 4 cycles, and based on follow-ups to date, the patient is in good condition.

\section{Discussion}

Currently, for locally advanced non-small cell lung cancer, radical concurrent chemoradiotherapy is still the main treatment mode, but its efficacy remains controversial due to serious side effects. For patients who can undergo surgery, neoadjuvant therapy combined with surgery can be a treatment option. Many neoadjuvant therapy modes, including targeted therapy, still need to be further studied. Current diagnosis and treatment guidelines encourage patients to participate in relevant clinical trials, and there have been some research reports on neoadjuvant targeted therapy.

The NCT01217619 study is a prospective single-arm phase II clinical study to evaluate the efficacy of erlotinib as a neoadjuvant therapy. A total of 19 patients with stage IIIA (N2) EGFR-mutated non-small cell lung cancer (NSCLC) were included, and 14 patients underwent surgery 8 weeks after beginning erlotinib treatment, of which 13 patients underwent successful radical resection. The proportion of postoperative pathological downstaging was $21.1 \%$, the objective response rate (ORR) was $42.1 \%$, and the disease control rate (DCR) was $89.5 \%$. After surgery, $50 \%$ of patients achieved a PR, and the remaining
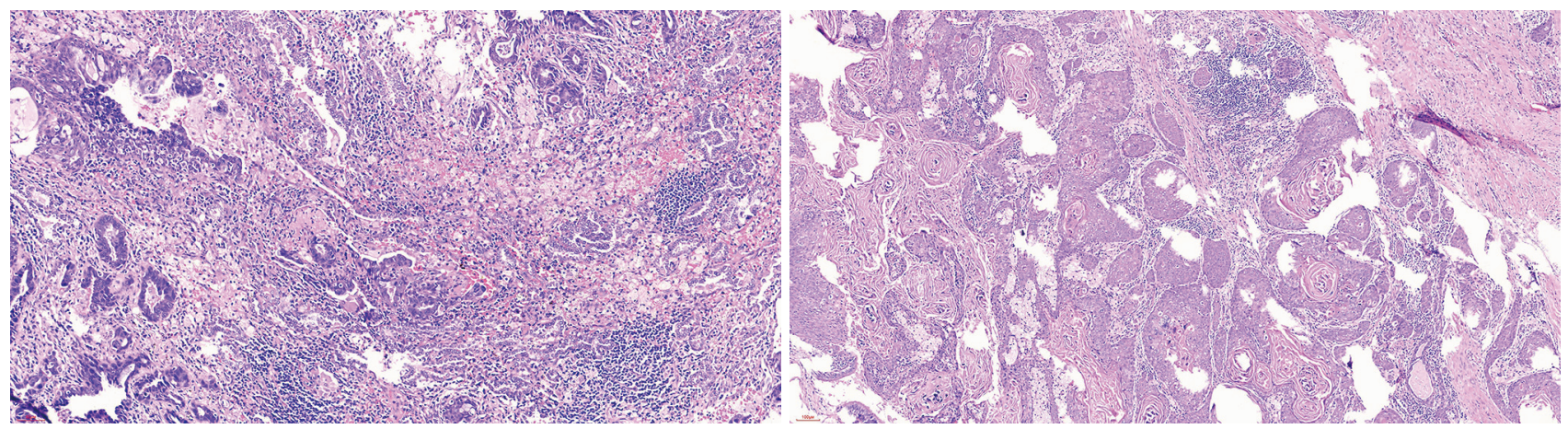

Figure 9 Postoperative pathology, adenocarcinoma component and squamous cell carcinoma component, HE staining, ×200. 
patients achieved stable disease (SD). The median diseasefree survival (DFS) of patients who underwent surgery was 10.3 months; among the 19 patients who received neoadjuvant therapy, the progression-free survival (PFS) and the median overall survival (OS) were 11.2 and 51.6 months, respectively (1). The NCT01833572 study is a prospective single-arm phase II clinical study to evaluate the efficacy of gefitinib as a neoadjuvant therapy. A total of 35 patients with stage II-IIIA EGFR-mutated NSCLC were included. After 5 years of follow-up, the ORR was $54.5 \%$, the MPR rate was $24.2 \%$, and the median DFS was 33.5 months. Patients with a MPR exhibited a significantly prolonged DFS $(\mathrm{P}=0.019)$, and there was a tendency to prolong OS (2). The EMERGING study (CTONG1103) is an open-label, randomized controlled clinical study conducted at 17 centers in China to evaluate the efficacy and safety of erlotinib versus platinum-based dual-drug therapy as a neoadjuvant therapy for stage IIIA (N2) NSCLC. A total of 72 patients received treatment, and the neoadjuvant ORRs for erlotinib and GC were $54.1 \%$ and $34.3 \%$, respectively $(\mathrm{P}=0.092)$. The proportions of patients with $\mathrm{R} 0$ resection and nodal downstaging were $73 \%$ and $10.8 \%$ vs. $63 \%$ and $2.9 \%$, in the erlotinib and GC groups, respectively. The PFS of the erlotinib and the GC chemotherapy groups were 21.5 and 11.4 months, respectively $(\mathrm{P}<0.001)$. In the GC group, $54.8 \%$ of patients were treated with second-line erlotinib after progression. The median OS of all patients was 32.5 months. Among the 55 surgical resection samples obtained, there was no pathologic complete response (PCR) in either group. However, $9.7 \%$ of patients in the erlotinib group achieved MPR, compared with $0 \%$ in the GC group. Pathological evaluations showed that at 27 months in the disease-free period, the proportion of lymphocyte infiltration was higher and tumor cells were significantly reduced after neoadjuvant erlotinib treatment (3). A phase II study of osimertinib as a neoadjuvant treatment for EGFRmutated NSCLC is ongoing, and data reported at the 2019 WCLC (World Conference On Lung Cancer) indicated that neoadjuvant osimertinib was well tolerated without significant surgical complications in the 6 patients initially treated. The MPR rate was $16 \%$ (1/6), the radiographic remission rate was $66 \%$ (4/6), and the DCR was $100 \%(6 / 6)$.

Some studies have also investigated postoperative tyrosine kinase inhibitor (TKI) adjuvant therapy. The ADJUVANT study, ADAURA study, and EVAN study all showed that $\mathrm{N} 2$ patients benefited more from postoperative adjuvant targeted therapy than from postoperative adjuvant chemotherapy $(4,5)$. Regarding the duration of postoperative TKI medication in patients with EGFR mutations, existing studies mostly use 2-year maintenance therapy (4-7), and no randomized controlled studies have provided an optimal maintenance medication time. For patients with stage IIIA NSCLC with EGFR mutations who received TKI adjuvant therapy, the role and timing of postoperative adjuvant radiotherapy are not clear.

The following lessons were learned from this case study. (I) The PET-CT for this patient revealed multiple metastases in the mediastinal lymph nodes. During the COVID-19 pandemic, our hospital was a designated treatment unit for COVID-19 patients in Fujian Province. Endobronchial ultrasound-guided transbronchial needle aspiration (EBUS-TBNA) was not performed for mediastinal lymph nodes for various reasons, which was unfortunate for this patient, and the possibility of a falsepositive PET-CT could not be completely ruled out. Fortunately, the patient's disease was significantly relieved after targeted therapy, and both the tumor and lymph nodes significantly decreased in size. Therefore, for N2 patients (suggested by imaging findings), biopsies should be performed to confirm the diagnosis and to facilitate a more accurate diagnosis and treatment. (II) For patients with squamous cell carcinoma who are clearly diagnosed using preoperative small specimens, if genetic testing suggests EGFR mutations, adenosquamous carcinoma should be considered. In particular, for patients with the EGFR L858R mutation, prioritizing the use of dacomitinib, icotinib + bevacizumab or icotinib may be beneficial. (III) It is worth exploring whether the timing of the surgical intervention was optimal for this case. The patient underwent surgery after 8 weeks of targeted therapy. The operation went smoothly, and there were only a few adhesions in the thoracic cavity. Would prolonged neoadjuvant therapy have aggravated the pleural and vascular inflammatory response and in turn increased the difficulty of surgery? Postoperative pathology showed that the lymph nodes were all negative and the tumor cells had decreased by approximately $30 \%$. Would prolonged neoadjuvant therapy have achieved MPR or even PCR? This patient had a relatively satisfactory condition during the treatment period; however, this patient constitutes only 1 case. Thus, with more cases, the observations herein can be further investigated.

Regarding treatment, it is feasible to perform surgery after neoadjuvant targeted therapy, and effective targeted therapy can downstage some locally advanced lung cancers, thereby allowing an opportunity for radical surgery and 
avoiding the pain of endless chemoradiotherapy. Surgery is helpful for identifying the molecular pathological changes in tumors after targeted therapy and can provide accurate information for subsequent individualized treatment. Neoadjuvant targeted therapy has few side effects and can improve the survival and quality of life of some patients. In conclusion, neoadjuvant targeted therapy is still in the exploration stage, and postoperative adjuvant therapy modes and prognoses need to be further studied. The emergence of more MPR and even PCR should be expected.

\section{Acknowledgments}

Funding: None.

\section{Footnote}

Reporting Checklist: The authors have completed the CARE Reporting Checklist. Available at http://dx.doi. org/10.21037/apm-21-868

Conflicts of Interest: All authors have completed the ICMJE uniform disclosure form (available at http://dx.doi. org/10.21037/apm-21-868). The authors have no conflicts of interest to declare.

Ethical Statement: The authors are accountable for all aspects of the work and for ensuring that questions related to the accuracy or integrity of any part of the work are appropriately investigated and resolved. All procedures performed in studies involving human participants were in accordance with the ethical standards of the institutional and/or national research committee(s) and with the Helsinki Declaration (as revised in 2013). Written informed consent was obtained from the patient for publication of this manuscript and any accompanying images.

Open Access Statement: This is an Open Access article distributed in accordance with the Creative Commons Attribution-NonCommercial-NoDerivs 4.0 International
License (CC BY-NC-ND 4.0), which permits the noncommercial replication and distribution of the article with the strict proviso that no changes or edits are made and the original work is properly cited (including links to both the formal publication through the relevant DOI and the license). See: https://creativecommons.org/licenses/by-nc-nd/4.0/.

\section{References}

1. Xiong L, Lou Y, Bai H, et al. Efficacy of erlotinib as neoadjuvant regimen in EGFR-mutant locally advanced non-small cell lung cancer patients. J Int Med Res 2020;48:300060519887275.

2. Zhang $\mathrm{Y}, \mathrm{Fu}$ F, Hu H, et al. Gefitinib as neoadjuvant therapy for resectable stage II-IIIA non-small cell lung cancer: A phase II study. J Thorac Cardiovasc Surg 2021;161:434-42.e2.

3. Zhong WZ, Chen KN, Chen C, et al. Erlotinib Versus Gemcitabine Plus Cisplatin as Neoadjuvant Treatment of Stage IIIA-N2 EGFR-Mutant Non-Small-Cell Lung Cancer (EMERGING-CTONG 1103): A Randomized Phase II Study. J Clin Oncol 2019;37:2235-45.

4. Zhong WZ, Wang Q, Mao WM, et al. Gefitinib versus vinorelbine plus cisplatin as adjuvant treatment for stage II-IIIA (N1-N2) EGFR-mutant NSCLC (ADJUVANT/ CTONG1104): a randomised, open-label, phase 3 study. Lancet Oncol 2018;19:139-48.

5. Yue D, Xu S, Wang Q, et al. Erlotinib versus vinorelbine plus cisplatin as adjuvant therapy in Chinese patients with stage IIIA EGFR mutation-positive non-small-cell lung cancer (EVAN): a randomised, open-label, phase 2 trial. Lancet Respir Med 2018;6:863-73.

6. Goss GD, O'Callaghan C, Lorimer I, et al. Gefitinib versus placebo in completely resected non-small-cell lung cancer: results of the NCIC CTG BR19 study. J Clin Oncol 2013;31:3320-6.

7. Kelly K, Altorki NK, Eberhardt WE, et al. Adjuvant Erlotinib Versus Placebo in Patients With Stage IBIIIA Non-Small-Cell Lung Cancer (RADIANT): A Randomized, Double-Blind, Phase III Trial. J Clin Oncol 2015;33:4007-14.
Cite this article as: Zhang N, Chen S, Guo L, Chen X. A case report of right upper lung adenosquamous carcinoma resection following neoadjuvant targeted therapy. Ann Palliat Med 2021;10(4):4987-4993. doi: 10.21037/apm-21-868 